\title{
Nonlinear fractional arctic systems
}

\author{
Sunday Christian Eze \\ Dept. of Mathematics, University of Nigeria, Nsukka, Nigeria \\ sundaychristianeze@gmail.com
}

\begin{abstract}
In this contribution, the nonlinear fractional arctic sea ice model is given, and the solution of the model was obtained using a new proposed modified Adomian decomposition method. The result is compared with the integer-order model, and we observed that a model with fractional order gives a better result. We also observed that the effect of climate change on arctic sea ice could lead to a large-scale sea ice melting with sea-level rise for several meters, which could pose a major threat to low-lying island nations and coastal areas.
\end{abstract}

Keywords: Adomian decomposition method; arctic sea ice; Caputo fractional derivative; carbon dioxide; climate change.

\section{Introduction}

The impact of anthropogenic climate change on arctic systems (such as mountain glaciers, Greenland ice sheets, and sea ice) is now well established as one of the most global threats to the human inhabitants, physical systems, and biological systems [see IPCC, (2001)]. The executive summary of the United Nations Intergovernmental Panel on Climate Change (IPCC, 2007) reported that climate change in arctic systems is now affecting the rest of the world by increasing the melting of glaciers, ice sheets, and sea ice that has an impact on the oceans.

As global temperatures rise, oceans get warmer and when water heats up, it expands, and sea levels rise. So, the melting of ice resting on the oceans does not increase the level of the oceans at the melting point of the ice, it is by a further increase in temperature due to the effect of anthropogenic greenhouse gases (GHGs) such as nitrous oxide $\left(\mathrm{N}_{2} \mathrm{O}\right)$, carbon dioxide $\left(\mathrm{CO}_{2}\right)$, methane $\left(\mathrm{CH}_{4}\right)$, which do not allow some solar radiation (or longwave radiation) to leave Earth's atmosphere, forcing it back to the Earth to be absorbed, causing global warming [Eze \& Oyesanya, (2019)].

In the work of Michael \& Thompson, (2011) and North, (1975), it was observed that to study the impact of climate change on arctic systems, it is better to start with mathematical modeling since it is the best starting point to test assumptions. Recently, various mathematical models have been developed to study the impact of climate change in arctic systems and we 
shall mention briefly a few works where more comprehensive references can be found. In the work of Walsh \& McGehee (2013), the energy balance models that provide the impact of climate change in glaciers were studied. Elizabeth et al. (2010) discussed the retrospective and the new direction of sea ice models. In the work of McGehee \& Lehman (2012), the model of paleoclimate ice albedo feedback forced by variations in Earth's orbit was discussed. Widiasih, (2013) discussed the Budyko-Sellers model when an ice sheet is allowed to respond to changes in temperature. The arctic systems are nonlinear [see Gre'millet (2015)] and can be modeled accurately by the nonlinear differential equation. This nonlinear differential equation is very important to scientists because it is the most valuable tool for modeling physical systems that have nonlinear structure interactions. But nonlinear differential equations hardly have exact analytical solutions. As a result, the approximate analytical or numerical methods such as Adomian decomposition method [see Adomian (1988) \& (1994)], modified Adomian decomposition method [see Khuri (2001) and (2004)], homotopy analysis method [see Ganjiana (2014)], variational iteration method [see He (2007) \& (1999)] and so on, have been developed for their approximate solutions.

In recent times, many researchers have demonstrated that nonlinear differential equations with fractional order derivatives are the most valuable tools for modeling physical phenomena that depend on current and previous time states. Among the researchers include (Eze \& Oyesanya, 2019), who used nonlinear fractional differential equation to study the impact of climate change in the pacific ocean, David \& Valentim(2015), who applied the Riemann Liouville approach and the fractional Euler-Lagrange equations to obtain nonlinear fractional differential equations of oscillatory systems, Eze(2020), who studied nonlinear Duffing oscillator using Caputo's fractional derivative operator, Ali et al.(2020), who examined the new wave solutions of an integrable dispersive wave equation with a fractional time derivative arising in ocean engineering models and Grzesikiewicz et al.(2013), who used fractional derivative operator to study nonlinear mechanical oscillator's problems.

The most widely used and very effective method for solving problems of fractional differential equations is the Laplace transform method (LTM). This LTM has been applied successfully by many researchers to solve various problems of linear fractional differential equations, which include the work of Li \& Peng (2019), Duffy (2004), Oyesanya (2015;2016), and Jumarie(2009). Despite this great success recorded by LTM to solve many problems of linear fractional differential equations, it has failed to solve nonlinear fractional differential equations because of the nonlinear terms. So, the applicability of this LTM to solve problems of nonlinear fractional differential equations shall be extended in this work.

This work is motivated by the desire to examine arctic systems (in particular, sea ice) using a fractional differential approach and to construct a new analytical method by modifying the Adomian decomposition method (ADM) in the spirit of power series for the analysis. Our emphasis will focus on the implications of higher emissions of $\mathrm{CO}_{2}$. 


\section{Basic definitions}

In this section, some basic definitions related to the Laplace transform (LT) and Caputo fractional derivative operator are given. Note that in this work, special attention will be paid to Caputo fractional derivative operator since in Caputo's definition; we can define initial conditions for fractional differential equations using the idea of the ordinary derivative operator.

Definition 2.1. The function $U(s)$ is called the LT of the original function $(\mathrm{t})$ and will be denoted by $L[u(t)]$. Thus:

$\mathcal{L}[u(t)]$

$=\int_{0}^{\infty} e^{-s t} u(t) d t$

Definition 2.2. The original function $u(t)$ in Definition 2.1 is called inverse transform or inverse of $U(s)$ and will be denoted by $\mathcal{L}^{-1}(U)$; that is, we shall write:

$u=\mathcal{L}^{-1}(U)$.

We can justify Equations (1) and (2) using the following two theorems:

Theorem 2.1. Let $u(t)$ be piecewise continuous on every finite interval in $t \geq 0$ and satisfy $|u(t)| \leq M e^{r t}$ for some constants $M$ and $r$. Then $\mathcal{L}[u(t)]$ exists for $s>r$ and

$$
\lim _{t \rightarrow \infty} \mathcal{L}[u(t)]=0
$$

Proof: It has to be shown that the LT of $u(t)$ is finite for $s>r$.

Advanced calculus implies that it is sufficient to show that the integrand is bounded above by integrable function $f(t)$.

Now, we take $f(t)=M e^{-(s-r)}$. Then, $f(t) \geq 0$. Furthermore, $f(t)$ is integrable since $\int_{0}^{\infty} f(t) d t=\frac{M}{s-r}$.

Inequality $|u(t)| \leq M e^{r t}$ implies that the absolute value of the $\operatorname{LT} \int_{0}^{\infty} e^{-s t} u(t) d t$ is estimated by $\left|u(t) e^{-s t}\right| \leq e^{r t} e^{-s t}=f(t)$.

The limit statement follows from $|u(t)| \leq \int_{0}^{\infty} f(t) d t=\frac{M}{s-r}$ because the right-hand side of this inequality has a limit zero at $s=\infty$. The proof is complete.

Theorem 2.2. If Equation (1) is satisfied by a continuous function, $u(t)$, there is no other continuous function that satisfies Equation (1). 
Proof: Now suppose that $f(t)$ is another continuous function satisfying Equation (1) and define $v(t)=u(t)-f(t)$ as a difference of two continuous functions, which is also continuous. Then $\int_{0}^{\infty} e^{-s t} u(t) d t=0$. Let $s=m+n$, wherem, $n \in \mathbb{Z}$. Then:

$\int_{0}^{\infty} e^{-(\mathrm{m}+\mathrm{n}) t} v(t) d t=\int_{0}^{\infty} e^{-\mathrm{n} t}\left[e^{-\mathrm{m} t} v(t)\right] d t=n \int_{0}^{\infty} e^{-\mathrm{n} t}\left[\int_{0}^{t} e^{-\mathrm{m} w} v(w) d w\right] d t$,

and thus it follows that $\int_{0}^{\infty} e^{-s t} v(t) d t=0$.

Hence, $\int_{0}^{\infty} e^{-s t} u(t) d t=\int_{0}^{\infty} e^{-s t} f(t) d t=0$.

Therefore, if we know the LT $U(s)$ of a function, we can recover the original function using the inverse LT of a function $\mathcal{L}^{-1}(U(s)) t$.

Remark: The LT is a linear operator and can only be applied to solve linear differential equations. Therefore, the applicability of this LT to solve problems of nonlinear differential equations using a new modified Adomian decomposition method in the spirit of power series shall be applied in this work.

Definition 2.3. For any functions $u(t)$ and $v(t)$ who's LT exists and any constant $a$ and $b$ we have

$\mathcal{L}[a u(t)+b v(t)]=a \mathcal{L}[u(t)]+b \mathcal{L}[v(t)]$.

Definition 2.4. The LT of a derivative with order $n \in \mathbb{N}$ for a function $u(t)$ is defined by

$$
\int_{0}^{\infty} e^{-s t}\left\{\frac{d^{n}}{d t^{n}} u(t)\right\} d t=s^{n} \mathcal{L}[u(t)]-\sum_{m=0}^{n} s^{n-m-1} u^{(m)}(0)
$$

Definition 2.5. The left sided Caputo fractional derivative with order $\alpha$ for a function $u(t)$ is defined by [see Podlubny (1990)]

$D_{t}^{\alpha} u(t)=\frac{1}{\Gamma(m-\alpha)} \int_{t_{0}}^{t}(t-\tau)^{m-\alpha-1} u^{m}(\tau) d \tau$,

Where, $D_{t}^{\alpha} u(t)=\frac{d^{\alpha}}{d t^{\alpha}} u(t), m-1 \leq \alpha<m, m \in \mathbb{N}$ and $t=t_{0}$ is the initial time and $\Gamma($.$) is the$ Gamma function.

Definition 2.6. The LT of the Caputo fractional derivative with order $\alpha$ is defined by [see Li \& Jiang (2014) and Go'mez-Aguilar et al. (2012)]

$$
\int_{0}^{\infty} e^{-s t}\left\{D_{t_{0}}^{\alpha} u(t)\right\} d t=s^{\alpha} U(s)-\sum_{m=0}^{n} s^{\alpha-m-1} u^{(m)}(0),
$$


Where, $n-1<\alpha \leq n, m, n \in \mathbb{N}$.

Definition 2.7. Two-parameter Mittag-Leffler function of $z$ is given by (Go'mez-Aguilar et al., 2012)

$E_{2 \alpha, \alpha}(z)$

$=\sum_{m=0}^{\infty} \frac{z^{m}}{\Gamma(2 \alpha m+\alpha)}$,

Where, $\alpha>0, m \in \mathbb{R}$ and $z \in \mathbb{C}$.

Definition 2.8. The LT of two-parameter Mittag-Leffler function is given by (Jumarie, 2009)

$$
\begin{aligned}
\mathcal{L}\left\{t^{2 \alpha m+\alpha-1} E_{2 \alpha, \alpha}^{m}\left( \pm \lambda t^{2 \alpha m}\right)\right\} & \\
= & \frac{m ! s^{\alpha-\beta}}{\left(s^{2 \alpha} \mp \lambda\right)^{m+1}},
\end{aligned}
$$

Where, $\alpha>0$ and $m, \lambda \in \mathbb{N}$.

\section{New modified ADM}

Let us present a new modification of ADM in the spirit of power series since it will provide a direct scheme to solve our models. For detailed information on ADM, see (Adomian, 1988 \& 1994) and references therein.

\subsection{New modified ADM for nonlinear initial value problems}

Now to propose a new modification of ADM for solving nonlinear initial value problems, we consider the following differential equation:

$$
\begin{aligned}
& \frac{d^{2} u(t)}{d t^{2}}+M u(t)+N u(t) \\
& =g(t),
\end{aligned}
$$

Subject to the initial conditions

$$
u(0)=a, \frac{d}{d t} u(0)=b,
$$

Where $M$ is a linear operator, $N$ is a nonlinear operator, $g$ is the inhomogeneous term, and $a, b \in$ $\mathbb{R}$ Applying LT on both sides of Equation (9) and using the differential property of LT [See Eq. (4)] with initial conditions Equation (10), we have: 


$$
\begin{aligned}
s^{2} \mathcal{L}[u(t)]+\mathcal{L}[M u(t)] \\
\quad=\mathcal{L}(g)+a s+b-\mathcal{L}[N u(t)] .
\end{aligned}
$$

Now we seek the power series solution of $u(t)$ in powers of $\eta$ in the form:

$$
\begin{aligned}
u(t)=\sum_{j=0}^{\infty} \eta^{j} & u_{j}(t) \\
& =u_{0}(t)+\eta u_{1}(t)+\eta^{2} u_{2}(t)+\cdots,
\end{aligned}
$$

Where, $\eta, \eta^{2}, \ldots$ are real constants, called the coefficients of the series.

Now, if we let $c_{j}=\eta^{j} u_{j}(t)$ in Equation (12), then the radius of convergence $\mathrm{R}$ can easily be obtained using ratio test as follows:

$$
\frac{1}{R}=\frac{c_{j}+1}{c_{j}}=\lim _{j \rightarrow \infty}\left|\frac{\eta^{j+1} u_{j+1}}{\eta^{j} u_{j}}\right| \Rightarrow \lim _{j \rightarrow \infty} \eta\left|\frac{u_{j+1}}{u_{j}}\right| .
$$

The ratio test says that the series converges if and only if $\frac{c_{j}+1}{c_{j}}<1$.

Therefore, $\lim _{j \rightarrow \infty}\left|\frac{c_{j}+1}{c_{j}}\right|<\frac{1}{\eta} \lim _{j \rightarrow \infty}\left|\frac{u_{j}}{u_{j+1}}\right|$.

Hence, the radius of convergence $R=\frac{1}{\eta}$. The interval of convergence is $I=\left(-\frac{1}{\eta}, \frac{1}{\eta}\right)$. If we let $\eta=1$, then the interval of convergence gives $I=(-1,1)$.

Therefore, the nonlinear term $N u(t)$ can now be decomposed as

$$
\begin{aligned}
& N u(t)=\sum_{j=0}^{\infty} \overline{A_{j}}\left(u_{0}, u_{1}, \quad u_{2}, \ldots,\right. \\
& \left.u_{j}\right),
\end{aligned}
$$

Where $\bar{A}_{j}$ is a new power series form of Adomian polynomial for the nonlinear term, say $N(u)=f(u)$, which is computed using the formula:

$$
\begin{aligned}
& \sum_{j=0}^{\infty} \bar{A}_{j} \\
& =\frac{1}{n !}\left[\frac{d^{n}}{d \lambda^{n}} N \sum_{j=0}^{\infty} \eta^{j+1} \lambda^{j} u_{j}(t)\right]_{\lambda=0},
\end{aligned}
$$

that is,

$$
\overline{A_{0}}=\eta f\left(u_{0}\right), \quad \overline{A_{1}}=\eta^{2} u_{1} f^{\prime}\left(u_{0}\right), \quad \overline{A_{2}}=\eta^{3}\left[u_{2} f^{\prime}\left(u_{0}\right)+\frac{1}{2 !} u_{1}^{2} f^{\prime \prime}\left(u_{0}\right)\right], \text { etc. }
$$


Note that $f^{n}\left(u_{0}\right)=\frac{f^{n}}{d u_{0}{ }^{n}}$.

Now we shall justify Equation (13) by using Taylor series expansion to construct the new Adomian polynomial for nonlinear term $N u(t)=f(u)$ and prove for it's convergent. Therefore, to construct a now Adomian polynomial (say $\bar{A}_{j}$ ) for $N=f(u)$, we define $\sum_{k=0}^{j} u_{j}$ and use Taylor series expansion about $u_{0}$ for the nonlinear term $f(u)$ to define $T_{j}$ as follows:

$$
\begin{gathered}
T_{0}=\eta f\left(u_{0}\right), T_{1}=\eta f\left(u_{0}\right)+\eta^{2}\left(S_{1}-S_{0}\right) f^{\prime}\left(u_{0}\right)+\eta^{3}\left(S_{1}-S_{0}\right)^{2} \frac{1}{2 !} f^{\prime \prime}\left(u_{0}\right) \\
T_{2}=\eta f\left(u_{0}\right)+\eta^{2}\left(S_{2}-S_{0}\right) f^{\prime}\left(u_{0}\right)+\eta^{3}\left(S_{2}-S_{0}\right)^{2} \frac{1}{2 !} f^{\prime \prime}\left(u_{0}\right)+\eta^{4}\left(S_{2}-S_{0}\right)^{3} \frac{1}{3 !} f^{\prime \prime \prime}\left(u_{0}\right), \\
T_{3}=\eta f\left(u_{0}\right)+\eta^{2}\left(S_{3}-S_{0}\right) f^{\prime}\left(u_{0}\right)+\eta^{3}\left(S_{3}-S_{0}\right)^{2} \frac{1}{2 !} f^{\prime \prime}\left(u_{0}\right)+\eta^{4}\left(S_{3}-S_{0}\right)^{3} \frac{1}{3 !} f^{\prime \prime \prime}\left(u_{0}\right) \\
+\eta^{5}\left(S_{3}-S_{0}\right)^{4} \frac{1}{4 !} f^{\prime \prime \prime \prime}\left(u_{0}\right), \\
\vdots T_{j}=\sum_{k=0}^{j+1} \eta^{k+1}\left(S_{n}-S_{0}\right)^{k} \frac{1}{k !} f^{(k)}\left(u_{0}\right), \quad j \geq 1 .
\end{gathered}
$$

Now, we can construct the new Adomain polynomial as follows:

Let define $\overline{A_{0}}=T_{0}=\eta f\left(u_{0}\right)$ and $\overline{A_{j}}=T_{j}-T_{j-1}, j \geq 1$.

Consequently,

$$
\begin{gathered}
\overline{A_{0}}=\eta f\left(u_{0}\right) \\
\overline{A_{1}}=\eta^{2} u_{1} f^{\prime}\left(u_{0}\right)+\eta^{2} \frac{1}{2 !} u_{1}^{2} f^{\prime \prime}\left(u_{0}\right) \\
\bar{A}_{2}=\eta^{3} u_{1} f^{\prime}\left(u_{0}\right)+\eta^{3}\left(u_{1} u_{2}+u_{2}^{2}\right) \frac{1}{2 !} f^{\prime \prime}\left(u_{0}\right)+\eta^{3}\left(u_{1}^{3}+3 u_{1}^{2} u_{2}+3 u_{1} u_{2}^{2}+u_{2}^{3}\right) \frac{1}{3 !} f^{\prime \prime \prime}\left(u_{0}\right) \\
\overline{A_{3}}=\eta^{4} u_{3} f^{\prime}\left(u_{0}\right)+\eta^{4}\left(2 u_{1} u_{3}+2 u_{2} u_{3}+u_{3}^{2}\right) \frac{1}{2 !} f^{\prime \prime}\left(u_{0}\right) \\
+\eta^{4}\left(3 u_{1}^{2} u_{3}+6 u_{1} u_{2} u_{3}+3 u_{2}^{2} u_{3}+3 u_{1} u_{3}^{2}+3 u_{2} u_{3}^{2}+u_{3}^{3} \frac{1}{3 !} f^{\prime \prime \prime}\left(u_{0}\right)\right) \\
+\eta^{4}\left(u_{1}+u_{2}+u_{3}\right)^{4} \frac{1}{4 !} u_{1}^{4} f^{\prime \prime \prime \prime}\left(u_{0}\right), \quad \text { etc. }
\end{gathered}
$$


Now for convergent, we take the infinity limit of $T_{j}=\lim _{n \rightarrow \infty} \sum_{k=0}^{j+1} \eta^{k+1}\left(S_{1}-S_{0}\right)^{k} \frac{1}{k !} f^{k}\left(u_{0}\right)$ to have:

$$
\begin{aligned}
\lim _{j \rightarrow \infty} T_{j}=\lim _{j \rightarrow \infty} \sum_{k=0}^{j+1}\left(S_{1}-S_{0}\right)^{k} \frac{1}{k !} f^{(k)}\left(u_{0}\right)=\sum_{k=0}^{\infty}\left(u_{j}-u_{0}\right)^{k} \frac{1}{k !} f^{(k)}\left(u_{0}\right) \\
\quad=f\left(u_{0}\right)+\left(u_{1}, u_{2},+\cdots\right) f^{\prime}\left(u_{0}\right) \\
+\left(u_{1}^{2}+2 u_{1} u_{2}+u_{2}^{2}+2 u_{1} u_{3}+2 u_{2} u_{3}+\cdots\right) \frac{1}{2 !} f^{\prime \prime}\left(u_{0}\right) \\
+\left(u_{1}^{3}+3 u_{1}^{2} u_{2}+3 u_{1} u_{2}^{2}+\cdots\right) \frac{1}{3 !} f^{\prime \prime \prime}\left(u_{0}\right) \\
+\left(u_{1}^{4}+4 u_{1}^{3} u_{2}+6 u_{1}^{2} u_{2}^{2}+\cdots\right) \frac{1}{4 !} f^{\prime \prime \prime \prime}\left(u_{0}\right)=\sum_{k=0}^{\infty} \bar{A}_{j} \\
=\eta f\left(u_{0}\right)+\eta^{2}\left(u-u_{0}\right) f^{\prime}\left(u_{0}\right)+\eta^{3}\left(u-u_{0}\right)^{2} \frac{1}{2 !} f^{\prime \prime}\left(u_{0}\right) \\
+\eta^{4}\left(u-u_{0}\right)^{3} \frac{1}{3 !} f^{\prime \prime \prime}\left(u_{0}\right)+\cdots,
\end{aligned}
$$

which is the Taylor series expansion for the nonlinear term $N=f(u)$ about the initial solution $u_{0}$.

$\lim _{j \rightarrow \infty} T_{j}=\lim _{j \rightarrow \infty} \sum_{j=0}^{j} \bar{A}_{j}=\sum_{j=0}^{\infty} \bar{A}_{j}=f\left(u_{0}\right)$.

Now since we have justified Eq. (13) and proved its convergent, then we substitute Equations (12) and (13) into Equation (11) to have:

$$
s^{2} \mathcal{L}\left[\sum_{j=0}^{\infty} \eta^{j} u_{j}(t)\right]+\mathcal{L}\left[M \sum_{j=0}^{\infty} \eta^{j} u_{j}(t)\right]=\mathcal{L}(g)+a s+b-\mathcal{L}\left[\sum_{j=0}^{\infty} \overline{A_{j}}\right] .
$$

Equating Equation (15) to the order of coefficients power of $\eta$, we have:

$$
\begin{aligned}
& \begin{array}{l}
\eta^{0}: s^{2} \mathcal{L}\left[u_{0}(t)\right]+\mathcal{L}\left[M u_{0}(t)\right] \\
=\mathcal{L}(g)+a s+b
\end{array} \\
& \begin{aligned}
\eta^{1}: & s^{2} \mathcal{L}\left[u_{1}(t)\right]+\mathcal{L}\left[M u_{1}(t)\right]=-\left[\mathcal{L}\left(A_{0}\right)\right],
\end{aligned} \\
& \eta^{2}: s^{2} \mathcal{L}\left[u_{2}(t)\right]+\mathcal{L}\left[M u_{2}(t)\right]=-\left[\mathcal{L}\left(A_{1}\right)\right], \\
& \eta^{3}: s^{2} \mathcal{L}\left[u_{3}(t)\right]+\mathcal{L}\left[M u_{3}(t)\right]=-\left[\mathcal{L}\left(A_{2}\right)\right], \\
& \text { etc. }
\end{aligned}
$$


where, $A_{0}=f\left(u_{0}\right), A_{1}=u_{1} f^{\prime}\left(u_{0}\right), A_{2}=u_{2} f^{\prime}\left(u_{0}\right)+\frac{1}{2 !} f^{\prime \prime}\left(u_{0}\right)$

Thus, the approximate solution of Eq. (9) with initial conditions Eq. (10) can now be obtained as:

$u(t)=\lim _{\eta \rightarrow 1}\left\{u_{0}(t)+\eta u_{1}(t)+\eta^{2} u_{2}(t)+\eta^{3} u_{3}(t)+\cdots\right\}$.

\subsection{New modified ADM for nonlinear fractional initial value problems}

Now to propose a new modification of ADM for solving nonlinear fractional initial value problems, we consider the following fractional differential equation:

$D_{t}^{\alpha} u(t)+M u(t)+F u(t)=g(t)$,

Subject to the initial conditions

$u(0)=c, \frac{d}{d t} u(0)=d$,

Where, $\alpha=\left[\alpha_{1}+\alpha_{2}, \ldots, \alpha_{n}\right]$ indicating fractional orders, $D_{t}^{\alpha}=\left[D_{t}^{\alpha_{1}}, D_{t}^{\alpha_{2}}, \ldots D_{t}^{\alpha_{n}}\right]$,

$1<\alpha_{i} \leq 2, i=1,2, \ldots, n, M$ is a linear operator, $F$ is a nonlinear operator, $g$ is the inhomogeneous term and $c, d \in \mathbb{R}$.

Applying LT on both sides of Equation (18) and using the fractional differential property of LT [see Eq. (6)] with initial conditions Equation (19), we have:

$s^{\alpha} \mathcal{L}[u(t)]+\mathcal{L}[M u(t)]=\mathcal{L}(g)+s^{\alpha-1} c+s^{\alpha-2} d-\mathcal{L}[F u(t)]$.

Now we seek the power series solution of $u(t)$ in powers of $\eta$ in the form:

$$
\begin{aligned}
u(t)=\sum_{j=0}^{\infty} \eta^{j} u_{j}(t) & \\
& =u_{0}(t)+\eta u_{1}(t)+\eta^{2} u_{2}(t)+\cdots,
\end{aligned}
$$

Where, $\eta^{0}, \eta^{1}, \eta^{2}, \ldots$ are real constants, called the coefficients of the series.

The nonlinear term $F u(t)$ is decomposed as

$$
\begin{aligned}
& F u(t)=\sum_{j=0}^{\infty} \overline{A_{j}}\left(u_{0}, u_{1}, \quad u_{2}, \ldots,\right. \\
& \left.u_{j}\right)
\end{aligned}
$$

Where, $\bar{A}_{j}$ is a new power series form of Adomian polynomial which is computed using the formula: 
$\sum_{j=0}^{\infty} \overline{A_{j}}=\frac{1}{n !}\left[\frac{d^{n}}{d \lambda^{n}} F \sum_{j=0}^{\infty} \eta^{j+1} \lambda^{j} u_{j}(t)\right]_{\lambda=0}$,

That, is, $\bar{A}_{0}=\eta f\left(u_{0}\right), \bar{A}_{1}=\eta^{2} u_{1} f^{\prime}\left(u_{0}\right), \bar{A}_{2}=\eta^{3}\left[u_{2} f^{\prime}\left(u_{0}\right)+\frac{1}{2 !} u_{1}^{2} f^{\prime \prime}\left(u_{0}\right)\right]$, etc.

Now, substituting Equations (21) and (22) into Equation (20), we have:

$$
s^{\alpha} \mathcal{L}\left[\sum_{j=0}^{\infty} \eta^{j} u_{j}(t)\right]+\mathcal{L}\left[M \sum_{j=0}^{\infty} \eta^{j} u_{j}(t)\right]=\mathcal{L}(g)+c s^{\alpha-1}+d s^{\alpha-2}-\mathcal{L}\left[\sum_{j=0}^{\infty} \bar{A}_{j}\right] .
$$

Equating Equation (24) to the order of coefficients power of $\eta$, we have:

$$
\begin{aligned}
\eta^{0}: s^{\alpha} \mathcal{L}\left[u_{0}(t)\right] & +\mathcal{L}\left[M u_{0}(t)\right] \\
& =\mathcal{L}(g)+c s^{\alpha-1}+d s^{\alpha-2}
\end{aligned}
$$

$\begin{aligned} \eta^{1}: s^{\alpha} \mathcal{L}\left[u_{1}(t)\right] & +\mathcal{L}\left[M u_{1}(t)\right] \\ & =-\left[\mathcal{L}\left(A_{0}\right)\right]\end{aligned}$

$\eta^{2}: s^{\alpha} \mathcal{L}\left[u_{2}(t)\right]+\mathcal{L}\left[M u_{2}(t)\right]=-\left[\mathcal{L}\left(A_{1}\right)\right]$,

$\eta^{3}: s^{\alpha} \mathcal{L}\left[u_{3}(t)\right]+\mathcal{L}\left[M u_{3}(t)\right]=-\left[\mathcal{L}\left(A_{2}\right)\right]$,

etc.

Thus, the approximate solution of Equation (18) with initial conditions Equation (19) can now be obtained as:

$u(t)=\lim _{\eta \rightarrow 1}\left\{u_{0}(t)+\eta u_{1}(t)+\eta^{2} u_{2}(t)+\eta^{3} u_{3}(t)+\cdots\right\}$.

\section{Sea ice model}

Now we shall build our model based on the models proposed by Saltzman (1978) to study the statistical-dynamical models of the terrestrial climate, modified in Saltzman et al. (1980, 1981a, 1981b \& 1982) and Nicolis (1993) \& (1982) to study the dynamic of climate feedback system involving sea ice extent and the variations in radiative heat input associated with variations in the orbit of the Earth respectively. These models were also studied by Bershadskii (2013) and Brindley et al. (1992) to analyze the interaction between the mass balance of the cryosphere and the surface energy balance.

Following Saltzman et al. and Brindley et al., the models describing the interaction between sea ice extent and temperature can now be written as: 


$$
\begin{aligned}
& \frac{d u(t)}{d t} \\
& =T(t)-u(t),
\end{aligned}
$$

$$
\begin{aligned}
\frac{d[T(t)-u(t)]}{d t} & \\
& =\zeta[T(t)-u(t)]+\kappa u(t)-u^{3}(t)-u^{2}(t)[T(t)-u(t)] \\
& +\sigma \sin \omega t,
\end{aligned}
$$

where $u(t)$ is the sea ice extent, $T(t)$ is the mean surface temperature, the constants $\zeta$ and $\kappa$ are the strengths of temperature and sea ice extent respectively, $\sigma$ sin $\omega t$ stands for solar forcing, $\sigma$ is the amplitude and $\omega$ is the frequency of the solar forcing.

Now differentiating Equation (26) with respect to $t$ gives:

$$
\begin{aligned}
& \frac{d^{2} u(t)}{d t^{2}} \\
& =\frac{d[T(t)-u(t)]}{d t} .
\end{aligned}
$$

Substituting Equations (26) and (28) into Equation (27), we have a model describing the interaction of arctic sea ice extent with respect to time as:

$$
\begin{gathered}
\frac{d^{2} u(t)}{d t^{2}}-\zeta \frac{d u(t)}{d t}+u^{2}(t) \frac{d u(t)}{d t}-\kappa u(t)+u^{3}(t) \\
=\sigma \sin \omega t .
\end{gathered}
$$

In 1896, Arrhenius (1896) proposed that the continuous combustion of fossil fuels may ultimately lead to enhanced global warming. In the combustion process, fuel reacts with oxidizing agents such as $\mathrm{CO}_{2}$ to yield $\mathrm{GHG}$ and energy as illustrated below:
I. $\mathrm{CH}_{4}+2 \mathrm{O}_{2} \rightarrow \mathrm{CO}_{2}+2 \mathrm{H}_{2} \mathrm{O}+$ Energy
II. $\mathrm{N}_{2}+2 \mathrm{O}_{2} \longrightarrow 2 \mathrm{NO}_{2}+$ Energy.

The warming on Earth is a result of the continuous combustion of fossil fuels which lead to GHGs such as $\mathrm{CO}_{2}$ and $\mathrm{NO}_{2}$ that trap longwave radiation in the atmosphere. The GHG that is of 
most public concern is $\mathrm{CO}_{2}$, a product of the burning of fossil fuels and associated organic matter.

$\mathrm{CO}_{2}$ is the largest contributor to global warming among all the anthropogenic GHGs [see Farmer \& Cook (2013)] and we shall consider its radiative forcing.

So, at a higher concentration of $\mathrm{CO}_{2}$, the value of radiative forcing $(\mathrm{R})$ can be expressed as [see Hermann (2017)]:

$R=\frac{T(C)-T(\hat{C}\})}{\mu} \frac{\operatorname{In} 2}{\operatorname{In}\left(\frac{C}{\tilde{C}}\right)}$,

where $\mu$ is the climate sensitivity parameter, which is assumed to take the estimated values between 1.2-11.8 [see Forster \& Gregory (2006)], T(C) is the temperature of $\mathrm{CO}_{2}, \hat{C}$ is the pre industrial concentration of $\mathrm{CO}_{2}$, which take the values from $275-280 \mathrm{ppm}$, and has continued to increase as a result of the continuous combustion of fossil fuels (see a Keeling curve in Figure 1 bellow, which showed an increase in a concentration of $\mathrm{CO}_{2}$ from $315 \mathrm{ppm}$ in 1958 to over 370ppm in 2004).

For a special case of Equation (30) where, $C=2 \hat{C}$, we have:

$$
\begin{aligned}
& R=\frac{T(C)-T(\hat{C}\})}{\mu} \\
& =\frac{\Delta T}{\mu},
\end{aligned}
$$

Which corresponds to the (IPCC, 2007) assessment report for the relationship between a sustained $\mathrm{R}$ and global mean surface temperature response $\Delta T$.

Therefore, taking the radiative forcing due to higher concentration of $\mathrm{CO}_{2}$ into consideration, Equation (29) now give:

$$
\begin{gathered}
\frac{d^{2} u(t)}{d t^{2}}-\zeta \frac{d u(t)}{d t}+u^{2}(t) \frac{d u(t)}{d t}-\kappa u(t)+u^{3}(t) \\
=\sigma \sin \omega t+R .
\end{gathered}
$$

Thus, the motion of the systems will be established by Equation (32) with the following initial conditions:

$u(0)=\frac{d}{d t} u(0)=0$ 


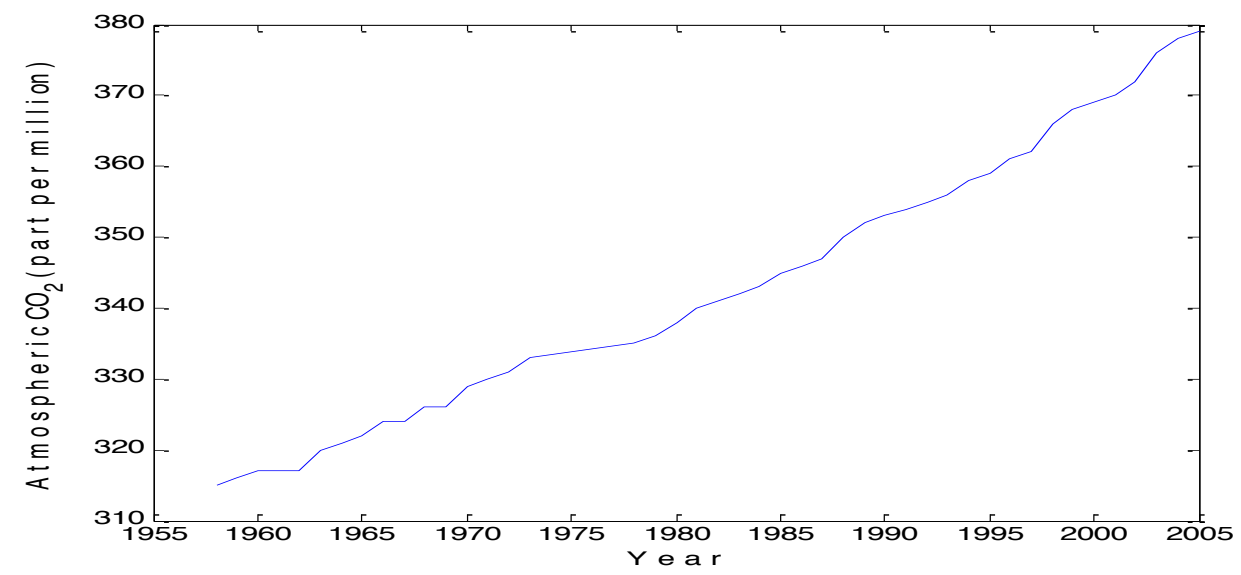

Fig. 1. The graph shows the concentration of atmospheric $\mathrm{CO}_{2}$ in the air at a weather station in Hawaii [Data source: Keeling et al. (2009)].

\section{Solution of the sea ice model}

New applying LT on both sides of Equation (32) and using the differential property of LT with initial conditions Equation (33), we have:

$s^{2} \mathcal{L}[u(t)]-s \zeta \mathcal{L}[u(t)]-\kappa \mathcal{L}[u(t)]=\frac{\sigma \omega}{s^{2}+\omega^{2}}+\frac{R}{s}-\mathcal{L}\left[u^{2}(t) \frac{d u(t)}{d t}+u^{3}(t)\right]$.

Now we seek the power series solution of $u(t)$ in powers of $\eta$ in the form:

$$
u(t)=\sum_{j=0}^{\infty} \eta^{j} u_{j}(t)=u_{0}(t)+\eta u_{1}(t)+\eta^{2} u_{2}(t)+\cdots
$$

The nonlinear term $u^{2}(t) \frac{d u(t)}{d t}+u^{3}(t)$ is decomposed as:

$$
\begin{gathered}
u^{2}(t) \frac{d u(t)}{d t}+u^{3}(t)=\sum_{j=0}^{\infty} \bar{A}_{j}\left(u_{0}, u_{1}, \quad u_{2}, \ldots,\right. \\
\left.u_{j}\right)
\end{gathered}
$$

Where $\bar{A}_{j}$ is a new power series form of Adomian polynomial for the nonlinear term, say $u^{2}(t) \frac{d u(t)}{d t}+u^{3}(t)=f(u)$, which is computed as follows: $\overline{A_{0}}=\eta f\left(u_{0}\right), \overline{A_{1}}=$ $\eta^{2} u_{1} f^{\prime}\left(u_{0}\right)$, etc.

Now, substituting Equations (35) and (36) in Equation (34) and equating to the order of coefficients power of $\eta$, we have: 


$$
\begin{aligned}
& \eta^{0}: \mathcal{L}\left[u_{0}\right] \\
& =\frac{1}{s^{2}-s \zeta-\kappa}\left[\frac{\sigma \omega}{s^{2}+\omega^{2}}\right. \\
& \left.+\frac{R}{s}\right] \\
& \eta^{1}: \mathcal{L}\left[u_{1}\right] \\
& =\frac{\mathcal{L}\left[A_{0}\right]}{s^{2}-s \zeta-\kappa}, \\
& \eta^{2}: \quad \mathcal{L}\left[u_{2}\right] \\
& =\frac{\mathcal{L}\left[A_{1}\right]}{s^{2}-s \zeta-\kappa},
\end{aligned}
$$

etc.

Let us now solve the sequence of equations for $u_{j}$.

To solve for $u_{0}$ we use Equation (35a), that is:

$$
\mathcal{L}\left[u_{0}\right]=\frac{1}{s^{2}-s \zeta-\kappa}\left[\frac{\sigma \omega}{s^{2}+\omega^{2}}+\frac{R}{s}\right] \Rightarrow u_{0}=\mathcal{L}^{-1}\left[\frac{\sigma \omega}{\left(s^{2}-s \zeta-\kappa\right)\left(s^{2}+\omega^{2}\right)}+\frac{R}{s\left(s^{2}-s \zeta-\kappa\right)}\right] .
$$

Now taking the partial fraction of each term, we have

$$
\begin{aligned}
& u_{0}=\mathcal{L}^{-1}\left\{\frac{\sigma \omega}{\omega^{4}\left(\zeta^{3}-\zeta\right)}\left[\frac{\omega^{2}-1}{s^{2}-s \zeta-\kappa}-\frac{\omega^{2}\left(\zeta+\zeta^{2}\right)}{s^{2}-s \zeta-\kappa}-\frac{\omega^{2}-1}{s^{2}+\omega^{2}}+\frac{\omega^{2} \zeta}{s^{2}+\omega^{2}}\right]\right. \\
& \left.-\frac{R}{\kappa}\left[\frac{1}{s}-\frac{s}{s^{2}-s \zeta-\kappa}-\frac{\zeta}{s^{2}-s \zeta-\kappa}\right]\right\} \\
& =\mathcal{L}^{-1}\left\{\frac{\sigma \omega}{\omega^{4}\left(\zeta^{3}-\zeta\right)}\left[\frac{\left(\omega^{2}-1\right)-\omega^{2}\left(\zeta+\zeta^{2}\right)}{s^{2}-s \zeta-\kappa}-\frac{\omega^{2}-1+\omega^{2} \zeta}{s^{2}+\omega^{2}}\right]\right. \\
& \left.-\frac{R}{\kappa}\left[\frac{1}{s}-\frac{s}{s^{2}-s \zeta-\kappa}-\frac{\zeta}{s^{2}-s \zeta-\kappa}\right]\right\} . \\
& \therefore u_{0}=\frac{\sigma \omega}{\omega^{4}\left(\zeta^{3}-\zeta\right)}\left[\left(\omega^{2}-1\right)-\omega^{2}\left(\zeta+\zeta^{2}\right)\left[\frac{2}{\sqrt{-4 \kappa-\zeta^{2}}} e^{-\frac{\zeta}{4} t} \sin \frac{\sqrt{-4 \kappa-\zeta^{2}}}{2} t\right]-\left(\omega^{2}-1\right.\right. \\
& \left.\left.+\omega^{2} \zeta\right) \sin \omega t\right]-\frac{R}{\kappa}\left[1-e^{-\frac{\zeta}{2} t} \cos \frac{\sqrt{-4 \kappa-\zeta^{2}}}{2}\right. \\
& \left.-\frac{\zeta}{\sqrt{-4 \kappa-\zeta^{2}}} e^{-\frac{\zeta}{4} t} \sin \frac{\sqrt{-4 \kappa-\zeta^{2}}}{2} t\right]
\end{aligned}
$$




$$
\begin{aligned}
& u_{1}=-\mathcal{L}^{-1}\left[\frac{\mathcal{L}\left(A_{0}\right)}{s^{2}-s \zeta-\kappa}\right], \\
& u_{2}=-\mathcal{L}^{-1}\left[\frac{\mathcal{L}\left(A_{1}\right)}{s^{2}-s \zeta-\kappa}\right],
\end{aligned}
$$

Where, $A_{0}=u_{0}^{2}(t) \frac{d}{d t} u_{0}+u_{0}^{3}$ and $A_{1}=2 u_{0} u_{1} \frac{d}{d t} u_{0}+u_{0}^{2} u_{0} \frac{d^{2}}{d t^{2}} u_{0}+3 u_{0}^{2} u_{1}$.

Thus, the solution of Equation (32) with initial conditions Equation (33) now gives:

$u(t)=u_{0}(t)+u_{1}(t)+u_{2}(t)+\ldots$

\section{Fractional Sea ice model}

Now we use the transformation operator in Rosales et al. (2011) and (Go'mez-Aguilar et al., 2012) to construct a corresponding fractional differential equation of Equation (32). The idea is to introduce an additional parameter $\delta$, which must have a dimension of time in seconds and be consistent with the dimension of the ordinary derivative operators. This $\delta$ represents fractional time components in the systems, and it is called cosmic time.

Given the above, we have:

$$
\begin{aligned}
& \frac{d}{d t} \rightarrow \frac{1}{\delta^{1-\alpha}} \frac{d^{\alpha}}{d t^{\alpha}} \\
& =\frac{1}{\delta^{1-\alpha}} D_{t}^{\alpha}, \\
& \frac{d^{2}}{d t^{2}} \rightarrow \frac{1}{\delta^{2-2 \alpha}} \frac{d^{2 \alpha}}{d t^{2 \alpha}} \\
& \quad=\frac{1}{\delta^{2-\alpha}} D_{t}^{2 \alpha},
\end{aligned}
$$

Where $0<\alpha \leq 1$.

Now applying Equations (39) and (40) in Equation (32), we have the fractional sea ice model as:

$$
\begin{gathered}
D_{t}^{2 \alpha} u(t)-2 \phi \omega_{0} D_{t}^{\alpha} u(t)+\rho u^{2}(t) D_{t}^{\alpha} u(t)-\omega_{0}^{2} u(t)+\rho^{2} u^{3}(t) \\
=\rho^{2} \sigma \sin \omega t+\rho^{2} R
\end{gathered}
$$

Where $\omega_{0}^{2}$ is the angular frequency of different values of $\alpha$, $\omega_{0}=\frac{\sqrt{\kappa}}{\delta^{\alpha-1}}, \rho=\frac{\omega_{0}}{\sqrt{\kappa}}$ and $\phi=\frac{\zeta}{2 \sqrt{\kappa}}$. 


\section{Solution of the fractional sea ice model}

Now applying LT on both sides of Equation (41) and using the fractional differential property of LT [see Eq. (6)] with initial conditions Equation (33), we have:

$$
\begin{aligned}
& s^{2 \alpha} \mathcal{L}[u(t)]-2 \phi \omega_{0} s^{\alpha} \mathcal{L}[u(t)]-\omega_{0}^{2} \mathcal{L}[u(t)] \\
& =\frac{\rho^{2} \sigma \omega}{s^{2}+\omega^{2}}+\frac{\rho^{2} R}{s}-\mathcal{L}\left[\rho^{2} u^{3}(t)\right. \\
& \left.+\rho u^{2}(t) D_{t}^{\alpha} u(t)\right] .
\end{aligned}
$$

Now we seek the power series solution of $u(t)$ in powers of $\eta$ in the form:

$$
u(t)=\sum_{j=0}^{\infty} \eta^{j} u_{j}(t)=u_{0}(t)+\eta u_{1}(t)+\eta^{2} u_{2}(t)+\cdots
$$

The nonlinear term $\rho^{2} u^{3}(t)+\rho u^{2}(t) D_{t}^{\alpha} u(t)$ is decomposed as:

$$
\begin{gathered}
\rho^{2} u^{3}(t)+\rho u^{2}(t) D_{t}^{\alpha} u(t)=\sum_{j=0}^{\infty} \bar{A}_{j}\left(u_{0}, u_{1}, \quad u_{2}, \ldots,\right. \\
\left.u_{j}\right)
\end{gathered}
$$

Where $\bar{A}_{j}$ is a new power series form of Adomian polynomial which is computed as follows:

$\bar{A}_{0}=\eta\left[\rho^{2} u_{0}^{3}(t)+\rho u_{0}^{2}(t) D_{t}^{\alpha} u_{0}(t)\right], \bar{A}_{1}=\eta^{2}\left\{3 \rho^{2} u_{0}^{2}(t)+2 \rho u_{0}(t) D_{t}^{\alpha} u_{0}(t) u_{1}(t)+\right.$ $\left.\rho u_{0}^{2}(t)\left[D_{t}^{\alpha} u_{0}(t)\right]^{\prime} u_{1}(t)\right\}$, etc.

Now, substituting Equations. (43) and (44) in Equation (42) and equating to the order of coefficients power of $\eta$, we have:

$$
\eta^{0}: \mathcal{L}\left[u_{0}(t)\right]=\frac{1}{s^{2 \alpha}-2 \phi \omega_{0} s^{\alpha}-\omega_{0}^{2}}\left[\frac{\rho^{2} \sigma \omega}{s^{2}+\omega^{2}}+\frac{\rho^{2} R}{s}\right]
$$

$$
\begin{aligned}
& \eta^{1}: \mathcal{L}\left[u_{1}(t)\right] \\
& =\frac{-\mathcal{L}\left[A_{0}\right]}{s^{2 \alpha}-2 \phi \omega_{0} s^{\alpha}-\omega_{0}^{2}},
\end{aligned}
$$




$$
\begin{aligned}
& \eta^{2}: \quad \mathcal{L}\left[u_{2}(t)\right] \\
& =\frac{-\mathcal{L}\left[A_{1}\right]}{s^{2 \alpha}-2 \phi \omega_{0} s^{\alpha}-\omega_{0}^{2}},
\end{aligned}
$$

etc.,

where

$$
\begin{aligned}
& u_{0}=\mathcal{L}^{-1}\left[\frac{1}{s^{2 \alpha}-2 \phi \omega_{0} s^{\alpha}-\omega_{0}^{2}}\left(\frac{\rho^{2} \sigma \omega}{s^{2}+\omega^{2}}+\frac{\rho^{2} R}{s}\right)\right], u_{1}=-\mathcal{L}^{-1}\left[\frac{\mathcal{L}\left(A_{0}\right)}{s^{2 \alpha}-2 \phi \omega_{0} s^{\alpha}-\omega_{0}^{2}}\right], \\
& u_{2}=-\mathcal{L}^{-1}\left[\frac{\mathcal{L}\left(A_{1}\right)}{s^{2 \alpha}-2 \phi \omega_{0} s^{\alpha}-\omega_{0}^{2}}\right], A_{0}=\rho^{2} u_{0}^{3}(t)+\rho u_{0}^{2}(t) D_{t}^{\alpha} u_{0}(t), A_{1}=3 \rho^{2} u_{0}^{2}(t)+ \\
& 2 \rho u_{0}(t) D_{t}^{\alpha} u_{0}(t) u_{1}(t)+\rho u_{0}^{2}(t)\left[D_{t}^{\alpha} u_{0}(t)\right]^{\prime} u_{1}(t) \text { and } u_{0}= \\
& \rho^{2} R \sum_{j=0}^{\infty}\left[\frac{\omega_{0}^{2 j}}{j !} t^{2 \alpha(j+1)} E_{\alpha, 2 \alpha+\alpha j+1}^{j}\left(2 \phi \omega_{0} t^{\alpha}\right)\right]+ \\
& \rho^{2} \sigma \sum_{k=0}^{\infty} \frac{(1)^{k}}{\omega^{2 k+1}} \sum_{j=0}^{\infty}\left[\frac{\omega_{0}^{2 j}}{j !} t^{2 \alpha(j+1)-2 k-1} E_{\alpha, 2 \alpha+\alpha j-2 k}^{j}\left(2 \phi \omega_{0} t^{\alpha}\right)\right] .
\end{aligned}
$$

Thus, the solution of Equation (41) with initial conditions Equation (33) now gives $u(t)=u_{0}(t)+u_{1}(t)+u_{2}(t)+\ldots$

\section{Discussion of results}

To predict and explain the impact of anthropogenic climate change in arctic systems, we generate the graphs of the solutions Equations (38) and (46) using the result of (PCC, 2007) assessment reports and the work of (Brindley et al., 1992).

In Figure 2, the oscillation is gradually decaying in amplitude, although not quite clear since the concentration of $\mathrm{CO}_{2}$ is low. In Figure 3, the oscillation is decaying very fast in amplitude as a result of a higher concentration of $\mathrm{CO}_{2}$. That means after a sufficiently long time, sea ice will melt to a very large scale, which could increase the average sea level rise to several meters and poses a major threat to low-lying island nations and coastal areas, meaning that these areas could be swamped and submerged by water anytime. The rise and fall of the sinusoidal curves in both Figures 2 and 3 is a result of the seasonal climate pattern of the arctic systems, which melt and shrink sea ice to its minimum extent around September and begin to increase during the winter and reaches its maximum extent in early March. Figure 4 showed that the fractional values $\alpha=3 / 4,1 / 2$, and $1 / 4$ exhibit a significant effect by showing clearly the decrease in sea ice extent, unlike Figure 2 which displayed only a sinusoidal curve. Figure 5 displayed expected behavior, 
that is, curves that are decreasing as a result of a higher concentration of $\mathrm{CO}_{2}$. Both Figures 4 and 5 showed that a good result is obtained at the smallest value of the fractional derivative. We, therefore, conclude that a better result is obtained with the fractional model.

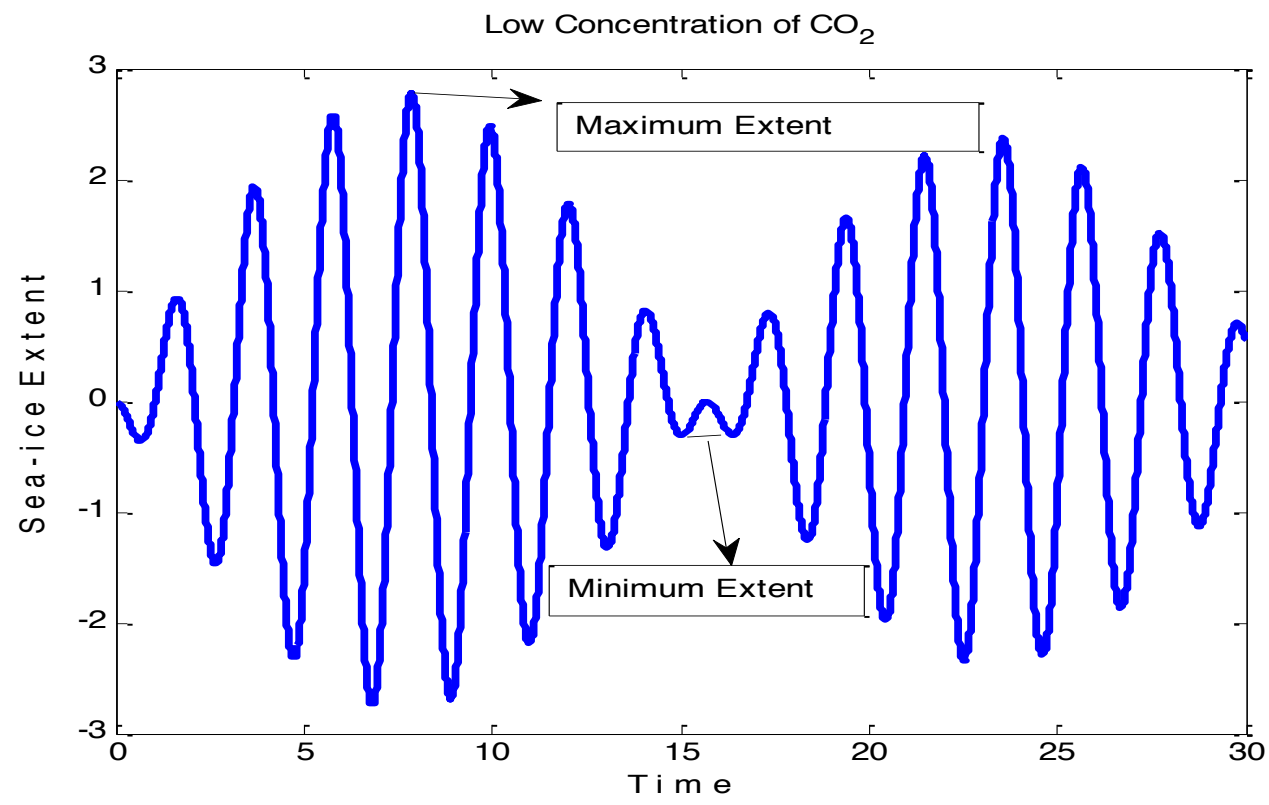

Fig. 2. Graph of Eq. (38), showing the impact of $\mathrm{CO}_{2}$.

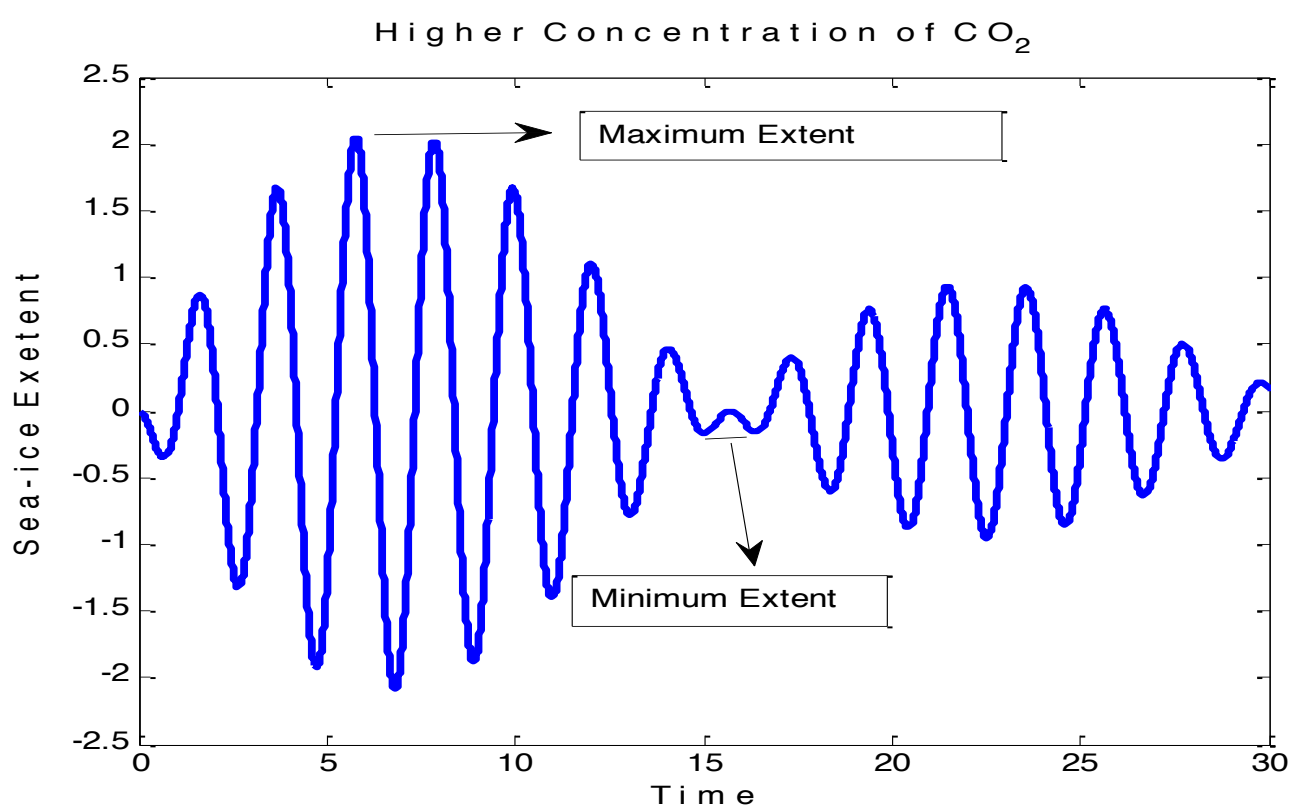

Fig. 3. Graph of Eq. (38), showing the impact of $\mathrm{CO}_{2}$. 


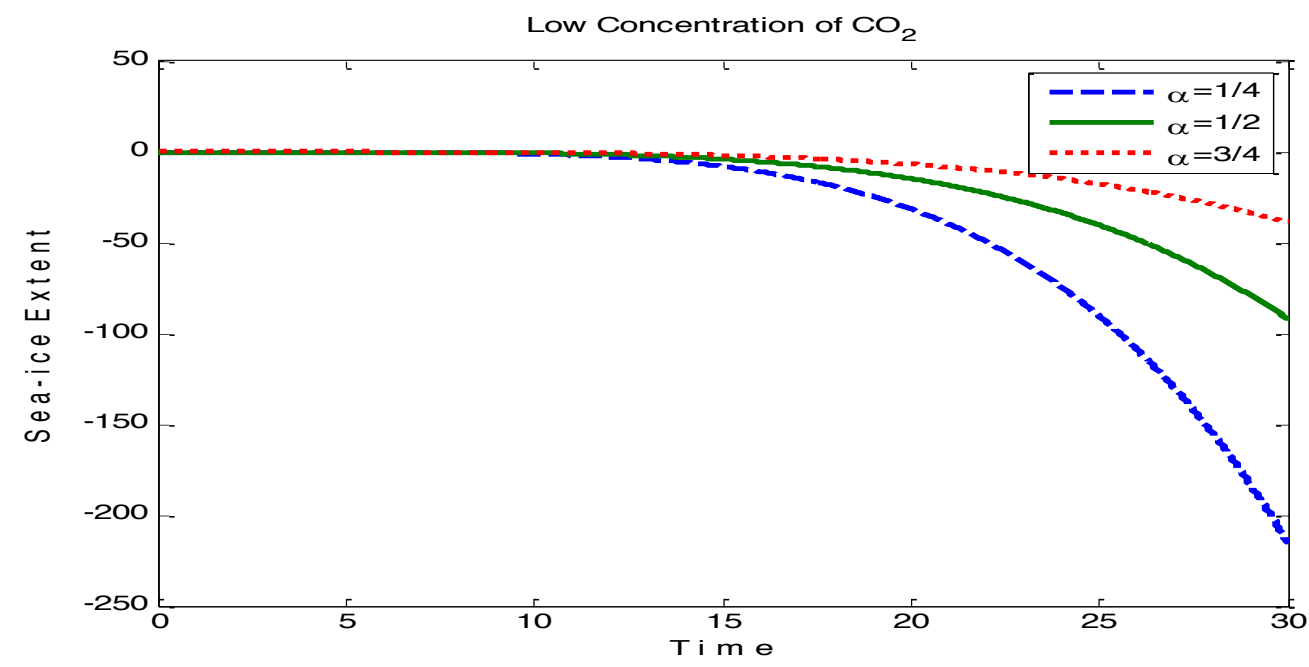

Fig.4. Graph of Eq. (46), showing the impact of $\mathrm{CO}_{2}$.

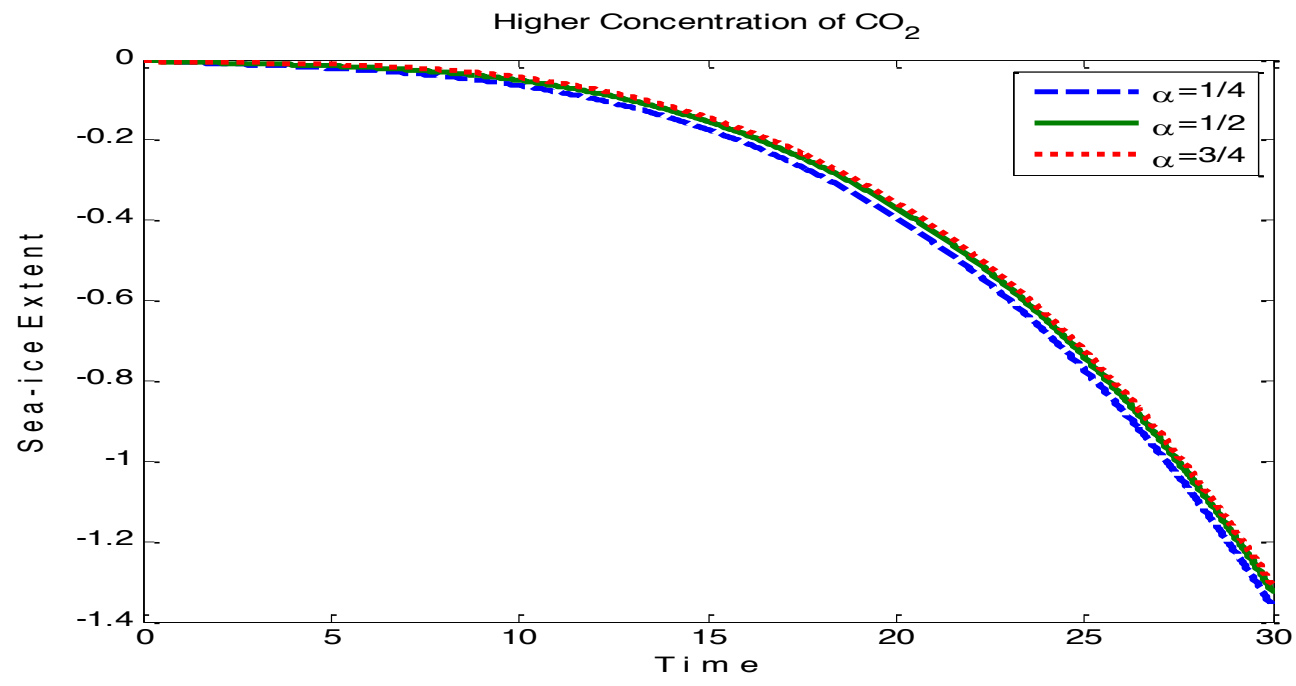

Fig. 5. Graph of Eq. (46), showing the impact of $\mathrm{CO}_{2}$.

\section{Conclusion}

The novelty of this work is on the mathematical formulation that further develops the climate model in arctic systems by capturing $\mathrm{CO}_{2}$ radiative forcing and fractional-order derivatives. This model helped to predict and explain the impact of anthropogenic climate change in arctic systems. The approximate solution of the model was obtained using a new proposed modified Adomian decomposition method. The result indicates that the impact of climate change on sea ice could increase the global sea-level rise and such an increase could cause flooding in many coastal areas, inducing saltwater intrusion into aquifers and submerging many coastal cities. 


\section{References}

Adomian, G. (1988). A review of the decomposition method in applied mathematics, Journal of Mathematical Analysis and Applications, 135: 501-544.

Adomian, G. (1994). Frontier problem of physics: The decomposition method, Boston: Kluwer Academic Publisher.

Ali, T. Ali, K. \& Orkun, T. (2020). New wave solutions of an integrable dispersive wave equation with a fractional time derivative arising in ocean engineering models, Kuwait Journal Science, 47 (2): 22-33.

Arrhenius, S. (1896). On the influence of carbonic acid in the air upon the temperature of the ground, Philosophical Magazine, 41: 237-276.

Bershadskii, A. (2013). Nonlinear problems of complex natural systems: sun and climate dynamics, Philosophical Transactions of the Royal Society A; 20120168.http://dx.doi.org/10.1098/rsta.2012.0168.

Brindley, J., Kapitaniak, T. \& Barcilon, A. (1992). Chaos and noisy periodicity in forced ocean-atmosphere models, Physics Letters A, 167: 179-184; DOI: 10.1016/03759601(92)90225-B.

David, S. A. \& Valentim, C.A.Jr. (2015). Fractional Euler-Lagrange equations applied to oscillatory systems, Mathematics, 3(2): 258-272.

Duffy D. G. (2004). Transform methods for solving partial differential equations, New York: Chapman and Hall/CRC;htts://doi.org/10.1201/9781420035148.

Elizabeth, C. H., William, H.L. \& Lipscomb K. T. (2010).Sea ice models for climate study: retrospective and new direction, Journal of Glaciology, 56:1162-1172.

Eze, S. C. \& Oyesanya, M. O. (2019). Fractional order on the impact of climate change with dominant earth's fluctuations, Mathematics of Climate and Weather Forecasting, 5: 1-11.

Eze, S.C. (2020). Analysis of fractional Duffing oscillator, Revista Mexicana de Fi'sica, 66 (2):187-191.

Farmer, G.T. \& Cook, J. (2013). Climate change science: a modern synthesis, Vol. 1: The physical climate, Dordrecht: Springer. 
Forster, P.M.D. \& Gregory, J.M. (2006). The climate sensitivity and its components diagnosed from Earth radiation budget data, Journal of Climate, 19: 39-52.

Ganjiana, M. (2014). Solution of nonlinear fractional differential equations using homotopy analysis method, Applied Mathematics, Modelling and Simulation, 36:1634-1641.

Go'mez-Aguilar, J. F., Rosales, J. J., Bernal-Alvarado, J. J., Cordova-Fraga, T. \& Guzman-Cabrera R. (2012). Fractional mechanical oscillators, Revista Mexicana de F''isica, 58: $348-352$.

Gre'millet, D. Fort, J. Ame'lineau, F. Zakharova, E. Le Bot, T. Sala, E \& Gavrilo, M. (2015). Arctic warming: Nonlinear impacts of sea ice and glacier melt on seabird forcing, Global Change Biology, 21(3): 1116-1123.

Grzesikiewicz, W., Wakulicz, A. \& Zbiciak, A. (2013). Nonlinear problems of fractional calculus in modeling of mathematical systems, International Journal of Mechanical Sciences, 70: 90-98.

He, J. (1999). Variational iteration method-a kind of nonlinear analytical technique: some examples, International Journal of Non-Linear Mechanics, 34(4): 699-708.

He, J. (2007). Variational iteration method-some recent results and new interpretations, Journal of Computational and Applied Mathematics, 207(1): 3-17.

Hermann, H. (2017). Radiation transfer calculations and assessment of global warming by $\mathrm{CO}_{2}$, International Journal of Atmospheric Sciences, (2017); https://doi.org/10.1155/2017/9251034.

IPCC, Climate Change 2001: Impacts, adaptation, and vulnerability, Contribution of Working Group II to the Third Assessment Report of the IPCC, McCarthy, James J. (eds.), Cambridge University Press.

IPCC, Climate Change 2007: The physical science basis, Contribution of Working Group I to the Fourth Assessment Report of the IPCC, Solomon. S et al. (eds.), Cambridge University Press.

Jumarie, G. (2009). Laplace's transform of fractional order via the Mittag-Leffler function and modified Riemann-Liouville derivative, Applied Mathematics Letters, 22:1659-1664.

Keeling, R.F., Piper, S.C., Bollenbacher A.F. \& Walker, J.S. (2009). Atmospheric $\mathrm{CO}_{2}$ records from sites in the SIO air sampling network. In trends: A compendium of data on global 
change. Carbon dioxide information analysis center, Oak Ridge National Laboratory, U.S. Department of Energy, Oak Ridge, Tenn., U.S.A.; DOI: 10.3334/CDIAC/atg.035.

Khuri, S. A. (2001). A Laplace decomposition algorithm applied to the class of nonlinear differential equations, Journal of Mathematical Analysis, 4: 141-155.

Khuri, S. A. (2004). A new approach to Bratu's problem, Applied Mathematics, and Computation, 147: 131-136.

Li, K. \& Peng, J. (2019). Laplace and fractional differential equations, Applied Mathematics Letters, 24: 2019-2023.

Li, X. \& Jiang, W. (2014). Solving fractional differential equations using the Laplace transform method, Abstract and Applied Analysis; https://doi.org/10.1155/2014/230850.

McGehee, R. \& Lehman, C. (2012). A paleoclimate model of ice-albedo feedback forced by variations in earth's orbit, SIAM Journal on Applied Dynamical Systems, 11(2): 684-707.

Michael, J. \& Thompson, T. (2011). Predicting climate tripping as a noisy bifurcation: A Review, International Journal of Bifurcation and Chaos in Applied Sciences and Engineering, 21(2): 399-423; DOI: 10.1142/50218127411028519.

Nicolis, C. (1982). Stochastic aspects of climate transitions-response to periodic forcing, Tellus, 34: $1-9$.

Nicolis, C. (1993). Long-term climatic transitions and stochastic resonance, Journal of Statistical Physics, 70: 3-14; DOI: 10.1007/BF01053950.

North, G. (1975). Analytical solution to a simple climate model with diffusive heat transport, Journal of the Atmospheric Sciences, 32 (1):1301-1307.

Oyesanya, M. O. (2015); (2016). Stability analysis of fractional Duffing oscillator, Transactions of the Nigerian Association of Mathematical Physics, 1: 133-150; 2: 325-342.

Podlubny, I. (1990). Fractional differential equations, New York, Academic Press.

Rosales, J. J., Go'mez, J. F., Guia, M. \& Tkach, V. I. (2011). In Proceedings of the 11 th International Conference on Laser and Fiber-Optical Networks Modeling, Kharkov, 2011, edited by I. A. Sukhoivanov and O. V. Shulika (IEEE, Kharkov, 2011), p. 1.

Saltzman, B. (1978). A survey of statistical-dynamical models of terrestrial climates, Advances in Geophysics, 20: 281-304. 
Saltzman, B. (1981a). Stochastically-driven climate fluctuations in the sea ice, ocean temperature, $\mathrm{CO}_{2}$ feedback system, Tellus, 34: 97-112.

Saltzman, B. \& Moritz, R.E. (1980). A time-dependent climate feedback system involving sea ice extent Ocean temperature and $\mathrm{CO}_{2}$, Tellus, 32: 93-118.

Saltzman, B., Sutera, A. \& Evenson, A. (1981b). Structural stochastic stability of a simple auto-oscillatory climate feedback system, Journal of the Atmospheric Sciences, 38: 494-503.

Saltzman, B., Sutera, A. \& Hansen, A. (1982). A possible marine mechanism for internally generated long-period climate cycles, Journal of the Atmospheric Sciences, 39: 2634-2637; DOI: 10.1175/1520-0469 039<2634: APMMFI >2.0.CO; 2.

Walsh, J. \& McGehee, R. (2013). Modeling climate dynamically. The College Mathematics Journal, 44(5): 350-363.

Widiasih, E. (2013). Dynamics of the Budyko-Sellers model, SIAM Journal on Applied Dynamical Systems, 12: 2068-2092.

Submitted: $22 / 11 / 2020$

Revised: 22/02/2021

Accepted: 09/03/2021

DOI: $10.48129 /$ kjs.v49i1.11145 